ELECTRONIC RESEARCH ANNOUNCEMENTS OF THE AMERICAN MATHEMATICAL SOCIETY

Volume 6, Pages 98-104 (December 7, 2000)

S $1079-6762(00) 00086-\mathrm{X}$

\title{
METRIC WITH ERGODIC GEODESIC FLOW IS COMPLETELY DETERMINED BY UNPARAMETERIZED GEODESICS
}

\author{
VLADIMIR S. MATVEEV AND PETAR J. TOPALOV
}

(Communicated by Dmitri Burago)

\begin{abstract}
Let $g$ be a Riemannian metric with ergodic geodesic flow. Then if some metric $\bar{g}$ has the same geodesics (regarded as unparameterized curves) with $g$, then the metrics are homothetic. If two metrics on a closed surface of genus greater than one have the same geodesics, then they are homothetic.
\end{abstract}

Let $g, \bar{g}$ be two $C^{2}$-smooth Riemannian metrics on a manifold $M^{n}$ of dimension $n \geq 2$. They are projectively equivalent if they have the same geodesics regarded as unparameterized curves. For a given metric $g$, there always exist trivial examples of projectively equivalent metrics: for any positive constant $C$, the metric $C g$ is projectively equivalent to $g$.

Consider the fiberwise-linear mapping $G: T M^{n} \rightarrow T M^{n}$ given by $G=g^{i \alpha} \bar{g}_{\alpha j}$ and the fiberwise-linear mapping $A: T M^{n} \rightarrow T M^{n}$ given by

$$
A \stackrel{\text { def }}{=}(\operatorname{det}(G))^{\frac{1}{n+1}} G^{-1} \text {. }
$$

Consider the characteristic polynomial $\operatorname{det}(A-\lambda \mathrm{Id})=c_{0} \lambda^{n}+c_{1} \lambda^{n-1}+\cdots+c_{n}$ and the mappings $S_{0}, S_{1}, \ldots, S_{n-1}: T M^{n} \rightarrow T M^{n}$ given by

$$
S_{n-m} \stackrel{\text { def }}{=} \sum_{i=0}^{m-1} c_{i} A^{m-i-1}
$$

Here $m$ lies in the set $\{1,2, \ldots, n\}$ so that $k=n-m$ lies in the set $\{0,1, \ldots, n-1\}$. Consider the functions $I_{0}, I_{1}, \ldots, I_{n-1}: T^{*} M^{n} \rightarrow R$ given by the general formula

$$
I_{k}(x, p) \stackrel{\text { def }}{=} g^{\alpha j}\left(S_{k}\right)_{\alpha}^{i} p_{i} p_{j} .
$$

In invariant terms, if we identify $T M^{n}$ with $T^{*} M^{n}$ via the metric $g$, the functions $I_{k}$ are given by $I_{k}(x, \xi)=g\left(S_{k} \xi, \xi\right)$.

Theorem 1 ([8]). If $g$ and $\bar{g}$ are projectively equivalent, then the functions $I_{k}$ are commutative integrals for the geodesic flow of the metric $g$.

If the geodesic flow of a metric is ergodic, any smooth integral is a function of the Hamiltonian and therefore only trivial examples of projective equivalence are possible. Basically we need not ergodicity but transitivity (= the existence of an

Received by the editors June 16, 2000 and, in revised form, October 1, 2000.

2000 Mathematics Subject Classification. Primary 53C20; Secondary 37J35, 37C40, 53A20, $53 \mathrm{C} 22,53 \mathrm{~B} 10$.

Key words and phrases. Projectively equivalent metrics, ergodic geodesic flows. 
orbit of the geodesic flow which goes arbitrary close to any point of the tangent bundle). By definition, almost any orbit of an ergodic geodesic flow is transitive.

Corollary 1. Let the geodesic flow of $g$ be ergodic. If some metric $\bar{g}$ is projectively equivalent to $g$, then $\bar{g}=C g$ for some constant $C$.

We will give a simple self-contained proof of Corollary 1 which does not use Theorem 1] Actually, in the proof of Corollary 1, we will demonstrate that the function $I_{0}$ is an integral for the geodesic flow of $g$. It is easy to see that the function $I_{0}$ is given by

$$
I_{0}(x, \xi)=-\left(\frac{\operatorname{det}(g)}{\operatorname{det}(\bar{g})}\right)^{\frac{2}{n+1}} \bar{g}(\xi, \xi) .
$$

More precisely, by Hamilton-Cayley theorem we have $c_{0} A^{n}+\cdots+c_{n} \equiv 0$. Then

$$
\begin{aligned}
S_{0} & =c_{0} A^{n-1}+c_{1} A^{n-2}+\cdots+c_{n-1} \\
& =A^{-1}\left(c_{0} A^{n}+\cdots+c_{n-1} A+c_{n}\right)-c_{n} A^{-1}=-\operatorname{det}(A) A^{-1} \\
& =-\left(\frac{\operatorname{det}(g)}{\operatorname{det}(\bar{g})}\right)^{\frac{2}{n+1}} G .
\end{aligned}
$$

The geodesic flow of a metric with negative sectional curvature on a closed manifold is ergodic; see the appendix by Brin in the book 1].

Corollary 2. Let $g$ be a Riemannian metric on $M^{n}$. Suppose that $M^{n}$ is closed, connected, and the sectional curvature of $g$ is negative. Then if some metric $\bar{g}$ is projectively equivalent to $g$, then $\bar{g}=C g$ for some constant $C$.

For closed surfaces, the integral of the curvature form over the surface is equal to the Euler characteristic. Then a metric with negative curvature can exist only on the surfaces of negative Euler characteristic. For such surfaces, we do not need the curvature restriction in Corollary 2 .

Corollary 3. Let $M^{2}$ be a closed connected surface of negative Euler characteristic, and let $g$ be a Riemannian metric on $M^{2}$. Then another Riemannian metric $\bar{g}$ on $M^{2}$ is projectively equivalent to $g$ if and only if $g=C \bar{g}$, where $C$ is a positive constant.

This corollary gives us a partial answer to the following classical question: for closed surfaces, what local structures constructed by a given metric, do not determine the metric locally, but determine the metric globally? Corollary 1 shows that, for surfaces of negative Euler characteristic, the projective class of a metric (= the set of all metrics projectively equivalent to the metric) uniquely defines the metric modulo multiplication by a constant.

The projective class of a metric is a locally defined object. It can be given in terms of the so-called projective connection (see for example Chapter 4 of Kobayashi [6]) or in terms of the Thomas vector field (see Thomas [10]).

Generally speaking, the projective class of a metric defines the metric neither locally nor on closed surfaces with Euler characteristic greater than or equal to zero. For the sphere, the first nontrivial examples of projectively equivalent metrics are due to Beltrami [2] for the torus such examples follow easily from Dini 4]. For the projective plane and for the Klein bottle such examples can be easily constructed using Beltrami's examples on the sphere and Dini's examples on the torus. 
Corollary 3 almost follows from Kolokol'tsov [7] or Kiyohara [5]. More precisely, the integral $I_{0}$ is quadratic in velocities so that the existence of a projectively equivalent metric implies the existence of an integral quadratic in velocities.

In [7] it was shown that the geodesic flow of a metric on a surface of negative Euler characteristic does not admit an integral which is

1. quadratic in velocities and

2. functionally independent of the Hamiltonian almost everywhere.

The second condition does not allow us to apply directly the result of [7] to our situation.

If a quadratic in velocities integral for the geodesic flow of a metric on a connected surface is functionally independent of the Hamiltonian at least at one point, then the integral is functionally independent of the Hamiltonian at almost every point. This fact was shown in [5] provided that the integral is not a linear combination of the Hamiltonian and the square of some linear in velocities integral; the latter condition does not allow us to apply directly the result of $[5]$ to our situation.

In our paper we give a simple self-contained proof of Corollary [3. The main construction is based on the idea of Birkhoff (see Chapter 2 of [3]) and has been used in [7].

Proof of Corollary 1 Let us show how the existence of $\bar{g}$ projectively equivalent to $g$ allows one to construct an integral for the geodesic flow of $g$. The construction is essentially due to [9] (see also [8]); it is slightly more general and allows one to construct an integral by an orbital diffeomorphism between two Hamiltonian systems.

Let $v$ and $\bar{v}$ be Hamiltonian systems on symplectic manifolds $\left(N^{2 n}, \omega\right)$ and $\left(\bar{N}^{2 n}, \bar{\omega}\right)$ with Hamiltonians $H$ and $\bar{H}$, respectively. Consider the isoenergy surfaces (which are not surfaces but submanifolds of codimension 1)

$$
Q^{2 n-1} \stackrel{\text { def }}{=}\left\{p \in N^{2 n}: H(x)=h\right\}, \quad \bar{Q}^{2 n-1} \stackrel{\text { def }}{=}\left\{p \in \bar{N}^{2 n}: \bar{H}(x)=\bar{h}\right\},
$$

where $h$ and $\bar{h}$ are regular values of the functions $H, \bar{H}$, respectively. A diffeomorphism $\phi: Q^{2 n-1} \longrightarrow \bar{Q}^{2 n-1}$ is orbital if it takes the orbits (regarded as unparameterized curves) of the system $v$ to the orbits of the system $\bar{v}$.

Given an orbital diffeomorphism, we can invariantly construct an integral for the system $v$. Denote by $\sigma, \bar{\sigma}$ the restrictions of the forms $\omega, \bar{\omega}$ to $Q^{2 n-1}, \bar{Q}^{2 n-1}$, respectively. Consider the pull-back $\phi^{*} \bar{\sigma}$, which is a skew-symmetric two-form on $Q^{2 n-1}$. By definition of the vector field $v$, for any vector field $u$ we have $\omega(v, u)=$ $d H(u)$. Then the kernel of the form $\sigma$ coincides (in the space $T_{q} Q^{2 n-1}$ at each point $q \in Q^{2 n-1}$ ) with the linear span of the vector $v$. Since the mapping $\phi$ takes orbits to orbits, the differential $d \phi$ takes the vector field $v$ to a vector field which is proportional to $\bar{v}$. Therefore the kernel of the form $\phi^{*} \bar{\sigma}$ also coincides (in the space $T_{q} Q^{2 n-1}$ at each point $q \in Q^{2 n-1}$ ) with the linear span of the vector $v$. Therefore the forms $\sigma, \phi^{*} \bar{\sigma}$ induce two nondegenerate two-forms on the quotient bundle $T Q^{2 n-1} /\langle v\rangle$. Recall that the quotient bundle $T Q^{2 n-1} /\langle v\rangle$ is the linear bundle over $Q^{2 n-1}$; the vectors of this bundle are equivalence classes of the vectors of $T Q^{2 n-1}$; for any $q \in Q^{2 n-1}$, two vectors $u_{1}, u_{2} \in T_{q} Q^{2 n-1}$ belong to the same class $U \in T_{q} Q^{2 n-1} /\langle v\rangle$ if and only if the vector $u_{1}-u_{2}$ is proportional to $v$. We will denote the corresponding forms on $T Q^{2 n-1} /\langle v\rangle$ also by $\sigma, \phi^{*} \bar{\sigma}$. Consider the fiberwise-linear mapping $\Sigma: T Q^{2 n-1} /\langle v\rangle \rightarrow T Q^{2 n-1} /\langle v\rangle$ uniquely determined by the condition $\sigma\left(\Sigma\left(U_{1}\right), U_{2}\right)=\phi^{*} \bar{\sigma}\left(U_{1}, U_{2}\right)$ for all $U_{1}, U_{2} \in T Q^{2 n-1} /\langle v\rangle$. Then the 
determinant $\operatorname{det}(\Sigma)$, which is a function on $Q^{2 n-1}$, is an integral of the system $v$. More precisely, by Cartan's formula, the Lie derivative $L_{v} \phi^{*} \bar{\sigma}$ of the form $\phi^{*} \bar{\sigma}$ on $Q^{2 n-1}$ along the vector field $v$ satisfies

$$
L_{v} \phi^{*} \bar{\sigma}=\mathrm{d}\left[\imath_{v} \phi^{*} \bar{\sigma}\right]+\imath_{v} \mathrm{~d}\left[\phi^{*} \bar{\sigma}\right] .
$$

On the right-hand side both terms vanish: the term $\mathrm{d}\left[\imath_{v} \phi^{*} \bar{\sigma}\right]$ vanishes since $v$ lies in the kernel of the form $\phi^{*} \bar{\sigma}$, and the term $\imath_{v} \mathrm{~d}\left[\phi^{*} \bar{\sigma}\right]$ vanishes since the form $\bar{\omega}$ is closed and therefore the form $\phi^{*} \bar{\sigma}$ is also closed. Hence the form $\phi^{*} \bar{\sigma}$ on $Q^{2 n-1}$ is preserved by the flow of $v$. It is known that the form $\omega$ and the Hamiltonian $H$ are also preserved by the flow of $v$. Therefore the form $\sigma$ and the vector field $v$ are preserved by the flow of $v$; hence the forms $\sigma, \phi^{*} \bar{\sigma}$ on $T Q /\langle v\rangle$ are also preserved by the flow of $v$ and finally the fiberwise-linear mapping $\Sigma$ and all its invariants, in particular the determinant, are preserved by the flow of $v$.

The geodesic flows of projectively equivalent metrics $g, \bar{g}$ on $M^{n}$ are orbitally equivalent systems on the symplectic manifolds $N^{2 n}=\bar{N}^{2 n}=T M^{n}$ with symplectic forms

$$
\omega \stackrel{\text { def }}{=} \mathrm{d}\left[g_{i j} \xi^{j} d x^{i}\right], \quad \bar{\omega} \stackrel{\text { def }}{=} \mathrm{d}\left[\bar{g}_{i j} \xi^{j} d x^{i}\right]
$$

and Hamiltonians $H, \bar{H}: T M^{n} \rightarrow R$ given by $H(x, \xi) \stackrel{\text { def }}{=} \frac{1}{2} g(\xi, \xi), \bar{H}(x, \xi) \stackrel{\text { def }}{=}$ $\frac{1}{2} \bar{g}(\xi, \xi)$. Here $(x, \xi) \in T M^{n}$ assuming that $x=\left(x^{1}, \ldots, x^{n}\right) \in M^{n}$ and $\xi=$ $\left(\xi^{1}, \ldots, \xi^{n}\right) \in T_{x} M^{n}$. The orbital diffeomorphism $\phi: T M^{n} \rightarrow T M^{n}$ is essentially the re-parameterization of geodesics, from the natural parameter of $g$ to the natural parameter of $\bar{g}$ :

$$
\phi(x, \xi) \stackrel{\text { def }}{=}\left(x, \frac{\|\xi\|_{g}}{\|\xi\|_{\bar{g}}} \xi\right)=\left(x, \frac{\sqrt{H}}{\sqrt{\bar{H}}} \xi\right) .
$$

The diffeomorphism $\phi$ obviously takes the isoenergy surface $Q^{2 n-1} \stackrel{\text { def }}{=}\{g(\xi, \xi)=1\}$ to the isoenergy surface $\bar{Q}^{2 n-1} \stackrel{\text { def }}{=}\{\bar{g}(\xi, \xi)=1\}$. Let us calculate the integral $\operatorname{det}(\Sigma)$ for these systems.

Consider the fiberwise-linear mapping $\Omega: T\left(T M^{n}\right) \rightarrow T\left(T M^{n}\right)$ uniquely determined by the condition $\omega\left(\Omega\left(u_{1}\right), u_{2}\right)=\phi^{*} \bar{\omega}\left(u_{1}, u_{2}\right)$ for any vectors $u_{1}, u_{2} \in$ $T\left(T M^{n}\right)$.

Let us first calculate the determinant of $\Omega$. The forms $\omega, \phi^{*} \bar{\omega}$ are given by

$$
\begin{aligned}
\omega= & d\left[\frac{\partial H}{\partial \xi^{i}} d x^{i}\right]=g_{i j} d \xi^{j} \wedge d x^{i}+\frac{\partial^{2} H}{\partial \xi^{i} \partial x^{j}} d x^{j} \wedge d x^{i}, \\
\phi^{*} \bar{\omega}= & d\left[\frac{\sqrt{H}}{\sqrt{\bar{H}}} \frac{\partial \bar{H}}{\partial \xi^{i}} d x^{i}\right]=\frac{\sqrt{H}}{\sqrt{\bar{H}}}\left(\bar{g}_{i j}+\frac{1}{2} \frac{\partial \bar{H}}{\partial \xi^{i}}\left(\frac{1}{H} \frac{\partial H}{\partial \xi^{j}}-\frac{1}{\bar{H}} \frac{\partial \bar{H}}{\partial \xi^{j}}\right)\right) d \xi^{j} \wedge d x^{i} \\
& +\frac{\partial^{2}\left(\frac{\sqrt{H}}{\sqrt{\bar{H}}}\right)}{\partial \xi^{i} \partial x^{j}} d x^{j} \wedge d x^{i} .
\end{aligned}
$$

We see that the formulae (2), (3) contain no element of type $d \xi^{i} \wedge d \xi^{j}$. Then in the coordinates $\left(x^{1}, \ldots, x^{n}, \xi^{1}, \ldots, \xi^{n}\right)$ the forms $\omega, \phi^{*} \bar{\omega}$ are given by $(2 n \times 2 n)$-matrices with zero lower right-hand side $(n \times n)$-blocks. Therefore, the determinant of $\Omega$ is equal, up to the sign $(-1)^{n}$, to the square of the ratio $\left(\frac{\operatorname{det}(a)}{\operatorname{det}(g)}\right)$, where $a$ is the 
two-form given by

$$
a_{i j} \stackrel{\text { def }}{=} \frac{\sqrt{H}}{\sqrt{\bar{H}}}\left(\bar{g}_{i j}+\frac{1}{2} \frac{\partial \bar{H}}{\partial \xi^{i}}\left(\frac{1}{H} \frac{\partial H}{\partial \xi^{j}}-\frac{1}{\bar{H}} \frac{\partial \bar{H}}{\partial \xi^{j}}\right)\right) .
$$

In coordinates, the matrix of the two-form $a$ is given by

$$
\frac{\sqrt{H}}{\sqrt{\bar{H}}} \overline{\mathbf{G}}(\mathbf{1}+\mathbf{P B})
$$

where $\overline{\mathbf{G}}$ denotes the matrix of the metric $\bar{g} ; \mathbf{1}$ denotes the identity matrix; the matrices $\mathbf{P}, \mathbf{B}$ are given by

$$
\mathbf{P}_{i j}=\frac{1}{2} \xi_{i} \xi_{j}, \quad \mathbf{B}_{i j}=\left(\frac{g_{i j}}{H}-\frac{\bar{g}_{i j}}{\bar{H}}\right) .
$$

Using that

$$
\sum_{i=1}^{n} \frac{1}{H} \frac{\partial H}{\partial \xi_{i}} \xi_{i}=\sum_{i=1}^{n} \frac{1}{\bar{H}} \frac{\partial \bar{H}}{\partial \xi_{i}} \xi_{i}=2
$$

we have $\mathbf{P B P}=0$ and therefore $(\mathbf{1}+\mathbf{P B})^{m}=(\mathbf{1}+m \mathbf{P B})$. Hence

$$
(\operatorname{det}(\mathbf{1}+\mathbf{P B}))^{m}=\operatorname{det}(\mathbf{1}+m \mathbf{P B}) .
$$

The left-hand side of (4) depends exponentially on $m$, whereas the right-hand side of (4) depends polynomially on $m$; it can happen only if $\operatorname{det}(\mathbf{1}+\mathbf{P B})=1$. Thus the determinant of the matrix of the form $a$ is equal to $\left(\frac{\sqrt{H}}{\sqrt{H}}\right)^{n} \operatorname{det}(\overline{\mathbf{G}})$ and the determinant of the fiberwise-linear mapping $\Omega$ is equal (up to the $\operatorname{sign}(-1)^{n}$ ) to $\left(\frac{\operatorname{det}(\bar{g})}{\operatorname{det}(g)}\right)^{2}\left(\frac{H}{H}\right)^{n}$.

Now let us prove that the determinants of $\Sigma$ and $\Omega$ are connected by

$$
\operatorname{det}(\Sigma)=\frac{H}{\bar{H}} \operatorname{det}(\Omega) \text {. }
$$

First of all, at each point $q \in Q^{2 n-1}$, the Hamiltonian vector field $v$ is an eigenvector of $\Omega$ with the eigenvalue $\frac{\sqrt{\bar{H}}}{\sqrt{H}}$. Indeed, it is easy to understand that $d \phi(v)=\frac{\sqrt{\bar{H}}}{\sqrt{H}} \bar{v}$ : the natural projection $\pi: T M^{n} \rightarrow M^{n}$ takes the vector $v$ to the vector of length one in the metric $g$ and takes the vector $\bar{v}$ to the vector of length one in the metric $\bar{g}$. Then for any $u \in T_{q} Q^{2 n-1}$ we have

$$
\begin{aligned}
\omega(\Omega(v), u) & =\phi^{*} \bar{\omega}(v, u)=\bar{\omega}(d \phi(v), d \phi(u)) \\
& =\frac{\sqrt{\bar{H}}}{\sqrt{H}} \bar{\omega}(v, d \phi(u))=\frac{\sqrt{\bar{H}}}{\sqrt{H}} d \bar{H}(d \phi(u)) .
\end{aligned}
$$

Since the mapping $\phi$ takes the $H$ to $\bar{H}$, then $\frac{\sqrt{H}}{\sqrt{H}} d \bar{H}(d \phi(u))=\frac{\sqrt{H}}{\sqrt{H}} d H(u)$ and therefore $\omega(\Omega(v), u)=\frac{\sqrt{H}}{\sqrt{H}} \omega(v, u)$. Thus $\Omega(v)=\frac{\sqrt{H}}{\sqrt{H}} v$.

Secondly, for any point $q \in Q^{2 n-1}$, the subspace $T_{q} Q^{2 n-1}$ of the tangent space $T_{q}\left(T M^{n}\right)$ is invariant under $\Omega$. Indeed, the necessary and sufficient condition for $u \in T_{q}\left(T M^{n}\right)$ to lie in $T_{q} Q^{2 n-1}$ is $\omega(u, v)=0$. Using that

$$
\omega(\Omega(u), v)=\omega(u, \Omega(v))=\frac{\sqrt{\bar{H}}}{\sqrt{H}} \omega(u, v)
$$

we obtain that if $u \in T_{q} Q^{2 n-1}$ then $\Omega(u) \in T_{q} Q^{2 n-1}$. 
Finally, since $v$ is an eigenvector of $\Omega$ and since $T_{q} Q^{2 n-1}$ is invariant under $\Omega$, any eigenvalue of $\Sigma$ is an eigenvalue of $\Omega$. The number of eigenvalues of $\Sigma$ (with multiplicities) is $2 n-2$; the number of eigenvalues of $\Omega$ (with multiplicities) is $2 n$; the two eigenvalues left are evidently the eigenvalue $\frac{\sqrt{H}}{\sqrt{H}}$ of the vector $v$ and the eigenvalue which corresponds to the eigenvector which does not lie in $T_{q} Q^{2 n-1}$. Using that the forms $\omega, \bar{\omega}, \sigma, \bar{\sigma}$ are skew-symmetric and therefore all eigenvalues of $\Omega, \Sigma$ have even multiplicities, we have that the eigenvalue of the eigenvector which does not lie in $T_{q} Q^{2 n-1}$ is also $\frac{\sqrt{H}}{\sqrt{H}}$. Using that the determinant is the product of all eigenvalues, we have $\frac{\bar{H}}{H} \operatorname{det}(\Sigma)=\operatorname{det}(\Omega)$ and therefore the function $\left(\frac{\operatorname{det}(\bar{g})}{\operatorname{det}(g)}\right)\left(\frac{H}{H}\right)^{n+1}$ is an integral for the geodesic flow of $g$. Using that the Hamiltonian $H$ is also an integral of the geodesic flow of $g$, we conclude that the function (1) is an integral for the geodesic flow of $g$.

Remark 1. Other characteristic invariants of the fiberwise-linear mapping $\Sigma$ give us the other integrals $I_{k}$.

Now suppose that the geodesic flow of the metric $g$ is transitive, in particular ergodic. Then any smooth integral must be a function of the Hamiltonian and the differentials of the functions $H$ and $I_{0}$ must be linear dependent at each point of $T M^{n}$. The partial derivatives $\frac{\partial H}{\partial \xi}, \frac{\partial I_{0}}{\partial \xi}$ are equal to

$$
\begin{aligned}
& \frac{\partial H}{\partial \xi}=\left(g_{1 j} \xi^{j}, g_{2 j} \xi^{j}, \ldots, g_{n j} \xi^{j}\right), \\
& \frac{\partial I_{0}}{\partial \xi}=\left(-2\left(\frac{\operatorname{det}(g)}{\operatorname{det}(\bar{g})}\right)^{\frac{2}{n+1}} \bar{g}_{1 j} \xi^{j},-2\left(\frac{\operatorname{det}(g)}{\operatorname{det}(\bar{g})}\right)^{\frac{2}{n+1}} \bar{g}_{2 j} \xi^{j}, \ldots,-2\left(\frac{\operatorname{det}(g)}{\operatorname{det}(\bar{g})}\right)^{\frac{2}{n+1}} \bar{g}_{n j} \xi^{j}\right) .
\end{aligned}
$$

Then the metrics must be at least conformally equivalent: $\bar{g}=f(x) g$. Let us prove that the conformal coefficient $f$ is constant. The integral $I_{0}$ for the pair of metrics $g, f(x) g$ is equal to $-\left(\frac{1}{f(x)^{n}}\right)^{\frac{2}{n+1}} f(x) g(\xi, \xi)=-\left(\frac{1}{f(x)}\right)^{\frac{n-1}{n+1}} g(\xi, \xi)$. Using that the Hamiltonian $\frac{1}{2} g(\xi, \xi)$ is an integral for the geodesic flow of $g$, we obtain that the function $\left(\frac{1}{f(x)}\right)^{\frac{n-1}{n+1}}$ is an integral for the geodesic flow of $g$. Joining any two points of $M^{n}$ by a geodesic, we see that $\left(\frac{1}{f(x)}\right)^{\frac{n-1}{n+1}}$ is a constant. Thus $\bar{g}=C g$. Corollary 1 is proved.

Proof of Corollary 3 Let $g, \bar{g}$ be projectively equivalent metrics on a closed surface $M^{2}$ of negative Euler characteristic. Without loss of generality we can assume that the surface $M^{2}$ is orientable. Consider the complex structure on $M^{2}$ corresponding to the metric $g$. Let $z$ be a complex coordinate in an open domain $U \subset M^{2}$, that is the metric $g$ reads $\lambda(z) d z d \bar{z}$, where $\lambda$ is a real-valued function. Consider the complex momentum $p$. In complex variables the Hamiltonian $H: T^{*} M^{2} \rightarrow R$ for the geodesic flow of the metric $g$ reads $2 \frac{p \bar{p}}{\lambda(z)}$. In complex coordinates, let the integral $F$ be given by $F=A(z) p^{2}+B(z) p \bar{p}+\bar{A}(z) \bar{p}^{2}$. Then $\frac{1}{A(z)} d z d z$ is a meromorphic two-form without zeros. More precisely, it is obviously a two-form: if we change holomorphically the coordinate system, then the coefficient $A$ changes as the reciprocal of the coefficient of a two-form. By definition, $\frac{1}{A}$ has no zero. We show that in each coordinate chart the function $A$ is holomorphic. Since $F$ is an integral of the Hamiltonian system with Hamiltonian $H$, the Poisson bracket 
$\{H, F\}$ equals zero. We have

$$
0=\{H, F\}=\frac{\partial H}{\partial p} \frac{\partial F}{\partial z}-\frac{\partial H}{\partial z} \frac{\partial F}{\partial p}+\frac{\partial H}{\partial \bar{p}} \frac{\partial F}{\partial \bar{z}}-\frac{\partial H}{\partial \bar{z}} \frac{\partial F}{\partial \bar{p}} .
$$

On the right-hand side of (4) each term is a polynomial of third degree in momenta. Then the bracket is also a polynomial of third degree in momenta. In order for the polynomial to equal zero, all coefficients must be zero, and in particular the coefficient of $p^{3}$. Thus $\frac{1}{\lambda} \frac{\partial A}{\partial \bar{z}}$ equals zero, and $A$ is holomorphic. Finally, $\frac{1}{A(z)} d z d z$ is a meromorphic two-form without zeros.

By Abel's theorem, for a meromorphic nontrivial two-form on a closed Riemann surface, the number of poles $P$ minus the number of zeros $Z$ is equal to twice the Euler characteristic. Then either the Euler characteristic of $M^{2}$ is negative or $A \equiv 0$. In the second case the metrics are conformally equivalent; in the proof of Theorem 1 we already showed that if two metrics are projectively equivalent and conformally equivalent, then the conformal coefficient is constant so that $\bar{g}=C g$. Corollary 3 is proved.

The authors are grateful to Professors Ursula Hamenstädt, Werner Ballmann, Gerhard Knieper, Anton Petrunin, Udo Simon, and Gudlaugur Thorbergsson for useful discussions. The first author thanks European Post-Doctoral Institute for financial support and Warwick University and Isaac Newton Institute for hospitality. The second author was partially supported by MESC grant MM-810/98 and by CNRS.

\section{REFERENCES}

[1] W. Ballmann, Lectures on spaces of nonpositive curvature. With an appendix by Misha Brin, DMV Seminar, 25. Birkhäuser Verlag, Basel, 1995. MR 97a:53053

[2] E. Beltrami, Resoluzione del problema: riportari i punti di una superficie sopra un piano in modo che le linee geodetische vengano rappresentante da linee rette, Ann. Mat. 1 (1865), no. 7 .

[3] G. D. Birkhoff, Dynamical Systems, Amer. Math. Soc. Colloq. Publ. 9, Amer. Math. Soc., New York, 1927. MR 35:1

[4] U. Dini, Sopra un problema che si presenta nella theoria generale delle rappresetazioni geografice di una superficie su un'altra, Ann. Mat., ser.2, 3 (1869), 269-293.

[5] K. Kiyohara, Compact Liouville surfaces, J. Math. Soc. Japan 43 (1991), 555-591. MR 92h:53056

[6] S. Kobayashi, Transformation groups in differential geometry, Classics in Mathematics. Springer-Verlag, Berlin, 1995. MR 96c:53040

[7] V. N. Kolokol'tzov, Geodesic flows on two-dimensional manifolds with an additional first integral that is polynomial with respect to velocities, Math. USSR-Izv. 21 (1983), no. 2, 291306. MR 84d:58064

[8] V. S. Matveev and P. J. Topalov, Trajectory equivalence and corresponding integrals, Regular and Chaotic Dynamics, 3 (1998), no. 2, 30-45. MR 2000d:37068

[9] P. J. Topalov, Tensor invariants of natural mechanical systems on compact surfaces, and the corresponding integrals, Sb. Math. 188 (1997), no. 1-2, 307-326. MR 98b:58136

[10] T. Y. Thomas, On the projective theory of two dimensional Riemann spaces, Proc. Nat. Acad. Sci. U. S. A. 31 (1945), 259-261. MR 7:33g

Isaac Newton Institute, Cambridge CB3 0EH, UK

E-mail address: v.matveev@newton.cam.ac.uk

Department of Differential Equations, Institute of Mathematics and Informatics, BAS, Acad. G. Bonchev Street, Bl. 8, Sofia 1113, Bulgaria

E-mail address: topalov@math.bas.bg 